\title{
Clinical efficacy of Qingchang Yuyang decoction combined with mesalazine in the treatment of ulcerative colitis
}

\author{
Song Jinxuan \\ School of Basic Medicine, North Sichuan Medical College, Clinical Medicine, Sichuan, China
}

Keywords: Ulcerative colitis, Qingchang Yuyang Decoction, Mesalazine, Clinical effect

\begin{abstract}
Aim: To observe the therapeutic effect of Qingchang Yuyang decoction combined with mesalazine on patients with ulcerative colitis. Methods: A total of 80 patients with ulcerative colitis admitted in a hospital in $2014 \sim 2017$ were selected. The patients were randomly divided into two groups: the experimental group and the control group, 40 cases in each group. The patients in the control group were treated with mesalazine granules alone and the patients in the experimental group were treated with Qingchang Yuyang decoction enema combined with mesalazine granules. Observe the clinical efficacy of two groups of patients, the treatment effect of different lesions, the improvement of clinical symptom score and the improvement of colonoscopy score ${ }^{[1]}$. Results: The experimental group was significantly better than the control group in clinical curative effect and clinical symptom score improvement, and the difference was statistically significant $(\mathrm{P}<0.05)$. Conclusion: The treatment of Qingchang Yuyang decoction combined with mesalazine for ulcerative colitis can effectively improve the therapeutic effect and the clinical symptoms of the patients. It is safe and reliable, and it is worthy of being widely used in clinical use.

Ulcerative colitis (UC) is a kind of chronic non-specific colon inflammation commonly seen in clinical practice. It has a long course of disease and high recurrence rate. Colonic mucosa and submucosa are the main lesions of the disease, which it mainly is ulcers. In severe cases, the rectum and distal colon often involve the proximal part of the colon and extend to the entire colon. The clinical manifestations of this disease are diarrhea, abdominal pain and mucous pus and blood stool $^{[2]}$. It has a greatly negative impact on the quality of life of the patient. According to the investigation, the incidence of ulcerative colitis in China has been increasing year by year, so we should focus on the more effective treatment of ulcerative colitis. According to the study, the treatment of the disease by western medicine alone did not obtain satisfactory curative effect, and the treatment of Qingchang Yuyang decoction combined with mesalazine could obviously improve the therapeutic effect. A group of 80 patients with ulcerative colitis were selected from a hospital to study the therapeutic effect of ulcerative colitis. The detailed reports are as follows:
\end{abstract}

\section{Data and methods}

\subsection{General information}

A total of 80 patients with ulcerative colitis treated in a hospital from 2014 to 2017 were selected and divided into two groups: experimental group and control group. There were 40 patients in the experimental group, 21 males and 19 females, with an age range of 27 69 years, with an average age of $(38.21+4.77)$ years. There were 40 patients in the control group, 18 male patients and 22 female patients, with an age range of $25 \sim 68$ years, with an average age of $(35.42+3.8 .7)$ years. The two groups had no statistical significance in sex, age and other basic conditions $(\mathrm{P}>0.05)$.

\subsection{Diagnostic criteria}

The two groups of patients were diagnosed accurately through the diagnosis and treatment of ulcerative colitis. (1) Inclusion criteria: (1) Compliance with diagnostic criteria. (2)Conforming to the diagnostic standard of 《Consensus in the diagnosis and treatment of inflammatory bowel disease in China $\rangle^{[3]}$. (3) Understand the research and voluntarily agree to participate in the study. (2) 
Exclusion criteria: (1) Patients during pregnancy and lactation, and those who are allergic to drugs.

(2) Patients suffering from colonic stenosis, intestinal obstruction, intestinal perforation, colon cancer, rectal cancer and other complications. Patients were not willing to participate in this study. (3)Having bad habits and failing to obey the doctor's advice.

\subsection{Methods}

The control group was given mesalazine granules 3 times /d, $1 \mathrm{~g} /$ times. The patients in the experimental group were treated with enema and enema based on the mesalazine granules. (Qingchang Yuyang soup: Pberetima, Cicada slough, Fructus Mume, Chebula 10g, Skullcap 15g, Pulsatilla 20g, water decoction two times mixed, make it concentrated to $200 \mathrm{ml}$.) Cool it to the right temperature and enema the patient every night. During the enema, the patient can get the proper position to ensure that the colon can be carried out smoothly. The drug is kept for at least 20 minutes.

\subsection{Observation Index}

\subsubsection{Clinical Efficacy}

According to 《Guidelines for clinical research on new drugs of traditional Chinese medicine (Implementation) ${ }^{[4]}$ the curative effect was determined. Markedly effective: All the clinical symptoms of the patients disappeared, the patients were observed to have smooth mucosa by enteroscopy, and no recurrence occurred within half a year after the patients stopped using the drugs; Effective: The clinical symptoms of the patients improved to some extent, but not all of them disappeared. A mild inflammatory reaction and partial polyp formation were observed in the patients by endoscopy; Ineffective: The clinical symptoms of the patients did not disappear, and no significant improvement was observed in the mucosa. The patient recurred many times within half a year after stopping medication. Total effective rate $=$ (Markedly effective + effective) $/$ number of patients * $100 \%$.

\subsubsection{Colonoscopy score criteria}

The colonoscopy results were divided into two groups: the patient's colon mucosa did not develop ulcers or edema. Erosion and hyperemia were recorded as 0 points; the number of ulcers in patients was less than 3 (including 3), and edema, erosion, and hyperemia were mild and were scored as 1; patients had more than 3 ulcers and edema and erosion. Congestion was more severe and was scored as 2 points; patients had more ulcers, edema, erosion, congestion, and bleeding at the ulcer site, scored as 3 points. The score is positively correlated with the severity of the patient's symptoms.

\subsubsection{Clinical symptom score criteria}

Dividing the clinical symptoms of the two groups of patients: (1)Diarrhea: 0 times per day, scored as 0 piont; occur no more than 3 times, scored as 1 piont; occur more than 3 times and less than 5 times, scored as 2 points. (2)Abdominal pain: No abdominal pain was scored as 0 point; slight pain was scored as 1 point; moderate pain was scored as 2 points. (3)Mucinous pus and bloody stool: No score was scored as 0 piont; a small amount was scored as 1 piont; medium amount was scored as 2 points.

\subsection{Statistical method}

SPSS20.0 statistical software was used for statistical analysis. Use $(\bar{x} \pm s)$ to represent the measurement data, use the $t$ test for the marker, use the rate (\%) to represent the count data, and use the $\mathrm{X}^{2}$ test. $\mathrm{P}<0.05$ indicates that the difference was statistically significant. 


\section{Results}

\subsection{Comparison of clinical efficacy between two groups of patients}

In the control group, 17 cases were markedly effective, 7 cases were effective, and 16 cases were invalid. The total effective rate was $60.0 \%$. In the experimental group, 25 patients were markedly effective, 8 patients were effective, and 7 patients were invalid. The total effective rate was $82.5 \%$. The total effective rate of the experimental group was significantly higher than that of the control group. The difference was statistically significant $(\mathrm{P}<0.05)$. See Table 1 for details

Table 1 Comparison of clinical efficacy and total effective rate between two groups of patients

\begin{tabular}{cccccc}
\hline Groups & Number of cases & $\begin{array}{c}\text { Markedly } \\
\text { effective }\end{array}$ & Effective & Invalid & $\begin{array}{c}\text { The total } \\
\text { effective rate }\end{array}$ \\
\hline Control group & 40 & $17(42.5 \%)$ & $7(17.5 \%)$ & $16(40.0 \%)$ & $60.0 \%$ \\
Experiment & 40 & $25(62.5 \%)$ & $8(20.0 \%)$ & $7(17.5 \%)$ & $82.5 \%$ \\
group & - & - & - & - & 3.905 \\
$X^{2}$ & - & - & - & - & $<0.05$ \\
P & & & & \\
\hline
\end{tabular}

\subsection{Changes in colonoscopy scores before and after treatment in both groups}

The colonoscopy scores were reduced in both groups of patients relative to treatment. The difference was statistically significant $(\mathrm{P}<0.05)$. After treatment, colonoscopy scores in the experimental group were significantly lower than in the control group. The difference was statistically significant $(\mathrm{P}<0.05)$. See Table 2 for details.

Table 2 Changes in colonoscopy scores before and after treatment in both groups

\begin{tabular}{cccc}
\hline Groups & Number of cases & $\begin{array}{c}\text { Colonoscopy score } \\
\text { before treatment }\end{array}$ & $\begin{array}{c}\text { Colonoscopy score } \\
\text { after treatment }\end{array}$ \\
\hline Experiment group & 40 & $1.8 \pm 0.7$ & $1.1 \pm 0.2$ \\
Control group & 40 & $1.9 \pm 0.6$ & $1.5 \pm 0.5$ \\
$\mathrm{t}$ & - & - & 6.644 \\
$\mathrm{P}$ & - & - & $<0.05$ \\
\hline
\end{tabular}

\subsection{Comparison of improvement of clinical symptoms before and after treatment in two groups of patients}

The proportion of the two groups was improved compared with the treatment before treatment. The difference was statistically significant $(\mathrm{P}<0.05)$. The degree of improvement in abdominal pain, diarrhea, and mucopurulent blood in the experimental group was significantly higher than that in the control group. The difference was statistically significant $(\mathrm{P}<0.05)$. See Table 3, Table 4 for details.

Table 3 Clinical symptom scores before treatment in both groups

\begin{tabular}{ccccc}
\hline Groups & Number of cases & Diarrhea & Abdominal pain & $\begin{array}{c}\text { Mucopurulent } \\
\text { blood }\end{array}$ \\
\hline Experiment group & 40 & $1.7 \pm 0.6$ & $1.9 \pm 0.7$ & $1.6 \pm 0.4$ \\
Control group & 40 & $1.8 \pm 0.7$ & $1.8 \pm 0.5$ & $1.8 \pm 0.9$ \\
\hline
\end{tabular}

Table 4 Clinical symptom scores after treatment in both groups

\begin{tabular}{ccccc}
\hline Groups & Number of cases & Diarrhea & Abdominal pain & $\begin{array}{c}\text { Mucopurulent } \\
\text { blood }\end{array}$ \\
\hline Experiment group & 40 & $1.2 \pm 0.4$ & $1.1 \pm 0.6$ & $1.3 \pm 0.2$ \\
Control group & 40 & $1.6 \pm 0.5$ & $1.4 \pm 0.6$ & $1.5 \pm 0.7$ \\
t & - & 5.587 & 3.162 & 2.457 \\
$\mathrm{P}$ & - & $<0.05$ & $<0.05$ & $<0.05$ \\
\hline
\end{tabular}




\section{Discussion}

Until now, the exact pathogenesis of ulcerative colitis has not been fully understood. However, after extensive clinical studies, it may be concluded that there may be 5 cases related to [5]: (1) Abnormal immune function. (2) Vascular expansion, increased permeability. (3) Food allergy induced. (4)Negative emotions. (5)Environmental or genetic factors. A relatively large number of studies have shown that ulcerative colitis is due to a certain induction of autoimmune responses due to genetic factors. After the onset of the disease, abdominal pain, diarrhea, and mucous pus and bloody stools may occur. Therefore, timely treatment should be performed after the onset of the patient.

For the treatment of ulcerative colitis, glucocorticoids and salicylates are mainly used for treatment. The mesalazine used in this study is a salicylic acid drug that can have a good effect on the inflammatory mediators of leukocytes, prevent the inflammatory reaction of the intestine, and relieve the patient's condition. However, due to the individual differences in patients, the use of mesalazine alone was not available to the patient and the expected efficacy was not obtained. In continuous trials, we found that Chinese medicine has a good effect on the intervention of ulcerative colitis, and there will be better healing effects for patients with colon cleansing. The Qingchang Yuyang Decoction is mainly for clearing away heat and dampness.

Its prescription Pulsatilla has detoxification and heat-clearing effect; Skullcap has the effect of dampness, heat, detoxification and clearing of the intestine; Cicada slough can disperse the wind to dispel heat; Pberetima can improve the patient's gastrointestinality. The microcirculation of the road. The drug can promote blood circulation, clear heat and dampness, speed up ulcer healing, and speed up mucosal regeneration ${ }^{[6]}$. The Qingchang Yuyang Decoction can directly reach the lesion, increase the concentration of the drug in the lesion, improve the local blood circulation, improve the immune function of the intestine, reduce the pain caused by the disease, and promote the absorption of inflammation. By enema to the patient, the digestive fluid can effectively avoid the destruction of the drug and the first-pass effect of the oral drug, thereby improving the utilization of the drug. The combination of Chinese and Western medicines can effectively exert their respective advantages and significantly improve the healing effect. Moreover, the formulation of traditional Chinese medicine is relatively mild and does not bring more stimulation to patients, which can reduce the occurrence of adverse reactions.

This study can be concluded that the treatment of ulcerative colitis with mesalazine, with the combination of clearing bowel soup and mesalazine, is obviously better than the treatment of salalazine alone. The total effective rate of the experimental group was $82.5 \%$, while that of the control group was $60 \%$. The experimental group was significantly higher than the control group. The difference was statistically significant $(\mathrm{P}<0.05)$. The edema, erosion and congestion of the patients in the experimental group were significantly improved. After treatment, the colonoscopy score in the experimental group was lower than that in the control group. The difference was statistically significant $(\mathrm{P}<0.05)$.

In conclusion, enema combined with oral mesalazine is effective in the treatment of ulcerative colitis. It has a positive effect on improving patients' quality of life and improving clinical symptoms. Drug use is relatively safe and reliable, with high drug utilization rate, and is worthy of wide use in clinical practice.

\section{References}

[1] Pan Zhuchun. Effect of Qing Chang Yu Yang Tang and mesalazine on ulcerative colitis. [J]. Oriental diet therapy and health care, 2017, (11): 284.

[2] Han Li. Qing Chang Yu Yang decoction combined with mesalazine in the treatment of ulcerative colitis [J]. Chinese medicine clinical research, 2017, (30): 75-76.

[3] Jin Xiang. Qing Chang Yu Yang decoction combined with mesalazine in the treatment of ulcerative colitis [J]. psychiatrist, 2016, (36): 141-142. 
[4] Zhang Juan, Cao Zewei. Qing Chang Yu Yang Tang combined with mesalazine in the treatment of ulcerative colitis [J]. Chinese general practice, 2012, (1): 90-92.

[5] Wang Jian. Qing Chang Yu Yang Tang combined with mesalazine in the treatment of ulcerative colitis: [J]. medicine frontier, 2013, (6): 186-187.

[6] Dang Zhongqin, Dang Zhibo, Wang Yuliang, etc. The clinical observation of 31 cases of chronic continuous ulcerative colitis with retention enema with Jianpi Qing Chang decoction combined with Yuyang enema in the treatment of chronic continuous ulcerative colitis, [J]. Chinese Journal of traditional Chinese medicine, 2015, (17): 1487-1490. 